\title{
PYCR2-related microcephaly-progressive leukoencephalopathy
}

INSERM

\section{Source}

INSERM. (1999). Orphanet: an online rare disease and orphan drug data base. PYCR2related microcephaly-progressive leukoencephalopathy. ORPHA:481152

PYCR2-related microcephaly-progressive leukoencephalopathy is a rare, genetic, syndromic intellectual disability disorder characterized by progressive postnatal microcephaly, cerebral hypomyelination and severe psychomotor developmental delayed with absent speech, as well as axial hypotonia, appendicular hypertonia with hyperextensibility of the wrists and ankles, hyperreflexia, severe muscle wasting and failure to thrive. Associated craniofacial dysmorphism includes triangular facies with bitemporal narrowing, down- or upslanting palpebral fissures, malar hypoplasia, large malformed ears with overfolded helices, upturned bulbous nose, long smooth philtrum and thin vermilion borders. 